\title{
Importance of Radiological Skeletal Assessment for Early Detection of Child Abuse
}

Aliaa Sait ${ }^{*}$, Muhannad Alnefaie ${ }^{2}$, Abdulaziz Aljohani ${ }^{3}$, Wafa Hadadi ${ }^{4}$, Maryiah Alhajji ${ }^{3}$, Mutlaq Alruwaili ${ }^{5}$, Maha Alshehri ${ }^{6}$, Hawraa Altaweel ${ }^{7}$, Mohammed Albaqshi ${ }^{8}$, Ameera Alhariqi ${ }^{9}$, Sarah Algosaibi ${ }^{10}$

${ }^{1}$ Department of Radiology, Al Aziziyah Children Hospital, Jeddah, Saudi Arabia

${ }^{2}$ Department of Pediatrics, Children Hospital, Taif, Saudi Arabia

${ }^{3}$ College of Medicine, Unaizah College of Medicine and Medical Sciences, Unaizah, Saudi Arabia

${ }^{4}$ College of Medicine, Jazan University, Jazan, Saudi Arabia

${ }^{5}$ College of Medicine, Al Jouf University, Sakaka, Saudi Arabia

${ }^{6}$ College of Medicine, King Khalid University, Abha, Saudi Arabia

${ }^{7}$ Department of Radiology, Prince Saud bin Jalawi Hospital, Al Ahsaa, Saudi Arabia

${ }^{8}$ College of Medicine, Medical University of Lublin, Poland

${ }^{9}$ College of Medicine, Princess Nourah Bint Abdulrahman Univeristy, Riyadh, Saudi Arabia

${ }^{10}$ Comprehensive Female Rehabilitation Center, Ministry of Human Resources and Social Development, Dammam, Saudi Arabia

Correspondence should be addressed to Aliaa Sait; Department of Radiology, Al Aziziyah Children Hospital, Jeddah, Saudi Arabia. Email: asait@moh.gov.sa

Received: 8 September 2021, Revised: 10 September 2021, Accepted: 12 September 2021, Published: 16 September 2021

Copyright (c) 2021 Sait et al. This is an open access article distributed under the Creative Commons Attribution License, which permits unrestricted use, distribution, and reproduction in any medium, provided the original work is properly cited.

\begin{abstract}
Evidence indicates that the physical abuse of children is a significant healthcare problem in various different communities. Therefore, eradicating this phenomenon can significantly enhance healthcare outcomes. To adequately diagnose the affected children, taking a full history and full examination of the child is essential to formulating a proper decision. In the present literature review, we have discussed the importance of radiological skeletal assessment in the early detection of child abuse. Conducting radiological skeletal surveys can play a significant role in detecting findings that can indicate the presence of physical abuse. X-ray, bone scan, and bedtime ultrasonography can be used to achieve this with variable sensitivities. However, X-ray remains the most sensitive, and the other two modalities should be used when performing X-ray is not appropriate. Two modalities can also be used at a time for better performance and enhanced outcomes. Imaging should be undertaken by at least one experienced radiologist for better interpretation of the findings. Finally, radiological skeletal surveys may be optimal for children of approximately two years of age, while in other children $\geq$ five years old, a case-bycase determination of assessment should be carried out.
\end{abstract}

Keywords: Radiological imaging, child abuse, diagnosis, skeletal survey. 


\section{Journal of Healthcare Sciences}

\section{Introduction}

Abusive injuries from caregivers that impact young children and infants are usually termed non-accidental injuries (1). Estimates indicate that such events are the most common causes of mortality and morbidity among children $(2,3)$. Increased risk of non-accidental injuries is usually highest in younger age groups, and caregivers are the main perpetrators of such injuries.

Evidence indicates that physical abuse of children represents a significant healthcare problem in a variety of communities (4). Therefore, eradicating this phenomenon can significantly enhance healthcare outcomes. To adequately diagnose affected children, taking a full history and full examination of the child is essential when formulating a proper decision (5). It has been demonstrated that radiological skeletal assessment is a critical tool for assessing and adequately diagnosing the physical abuse of children. Accordingly, the radiologist plays an important role in these situations as their expertise can differentiate between the physical abuse of a child and findings that are attributable to other etiologies. The radiologist can also suggest the mechanism of injury, which may be important for legal purposes. In the present literature review, we aim to discuss the importance of a radiological skeletal survey for the early detection of child abuse.

\section{Review}

Studies in the literature have demonstrated the importance and value of radiological skeletal assessment surveys for the early detection of child abuse events. Evidence indicates that whenever child physical abuse has been suspected in children $\geq 2$ years old, performing radiological skeletal surveys should be mandatory. It should also be performed for older children with verbal difficulties and developmental delays as these children usually have difficulties with reporting, and therefore, it can be hard to clinically assess them. A further important indicator for conducting radiological skeletal surveys could be when that child abuse is detected in children, their siblings should also be assessed for suspected abuse (6). Evidence indicates that utilizing radiological skeletal surveys is usually limited to children $>2$ years old, and it has been demonstrated that the modality is of limited value beyond the age of 5 years old. In a previous review, the authors reported that the findings of physical abuse and skeletal injuries were similar on the radiological skeletal surveys performed for 24-36 and 12-24 month-old children (7). It has also been suggested that a low threshold for 3-year-old children should be utilized for a radiological skeletal survey. Therefore, determining the value of conducting radiological skeletal surveys for children that are $>5$ years of age should be considered on a case-by-case basis (8). The modality has been recommended as the first-line approach in cases where child abuse is suspected and in such cases, whole skeletal imaging should to adequately establish a proper diagnosis.

The Society for Pediatric Radiology, together with the American College of Radiology, have previously collaborated to establish proper and consistent guidelines to adequately conduct a radiological skeletal assessment for children that are suspected of being exposed to child abuse (9). Compelling evidence of the presence of a skeletal injury and the adequate diagnosis of child abuse can be obtained from skeletal surveys, although it has been previously demonstrated that skeletal injuries are not usually life-threatening. Identification of the patterns of the skeletal injury can be obtained by conducting relevant skeletal surveys, and some of the potential findings may include posterior rib fractures, and corner or bucket-handle fractures, which refer to long bones-related classical metaphyseal lesions (10-12). These two findings have been reported to be significant indicators of the presence of underlying child abuse and significantly add to the diagnostic value when other clinical data are missing. Performing radiological skeletal surveys can identify the timing of the injury and can also examine and quantify the perpetrators of abuse, in addition to helping with the identification of whether caregivers were innocent or perpetrators of abusive behavior. It should also be noted that the findings of the radiological skeletal surveys can also be demanded for legal proceedings to providing a complete picture of the underlying cause (13). As a result, those performing radiological skeletal surveys should be careful to conduct the survey properly to adequately identify any underlying pathology and help establish an accurate diagnosis. This can be done by using rigorous protocols and imaging systems with high-detailed qualities, in addition to providing adequate consideration to the conning and centralizing of the imaging technique according to the targeted body parts and shading of other parts. Adequate attention should also be given to the position of the patient using restraining modalities (14-16). High-quality images of the targeted skeletal parts can be easily obtained using these approaches, in addition to minimizing the degree 


\section{Journal of Healthcare Sciences}

of radiation exposure to a reasonable level that does not affect the targeted findings (9). Every different anatomical region should be captured in a single image, although it has also been suggested that some anatomical regions may require more than one imaging projection for easy interpretation of the findings and a proper diagnosis. For instance, it has been previously demonstrated that to successfully detect suspected rib fractures, combining the left and right oblique views of the thorax is recommended as a valid imaging protocol (9). Evidence indicates that rib fractures are important parameters that can help identify a potential underlying trauma both to adults and adolescents. However, it is known that deformities can form before fractures occur in younger children who have been exposed to a traumatic event Accordingly, the ability to detect rib fractures in children has been demonstrated to be very limited because of the plasticity of their ribcage. It has also been demonstrated that rib fractures can be accidentally identified as an occult trauma (17). Therefore, studies have indicated that detecting rib fractures at an early age is a significant indicator of the presence of trauma secondary to child abuse (18). Antero-posterior compression of the chest is also an important mechanism that can lead to rib fractures $(19,20)$. In a previous investigation by Barsness et al. (21), the authors evaluated the positive predictive value of detecting child abuse based on the radiological skeletal assessment of rib fractures. The authors indicated that the positive predictive value for diagnosing child abuse was $95 \%$, which has been validated on 316 rib fractures among 62 children $<3$ years old. A further investigation also indicated that rib fractures usually require stronger forces to induce fractures in children than those that may induce fractures for older age groups (22). Although it has been demonstrated that evaluation of a potentially underlying medical condition (including metabolic diseases and skeletal disorders) might exhibit a wider imaging view of the skeleton and can be associated with better imaging modalities, and can also be theoretically more valuable than conducting skeletal surveys, however, it should be noted that the role of "babygram" in these situations is limited concerning the diagnosis of child abuse $(8,9)$.

A radiologist must be present at the time of interpretation of the radiological images to adequately report the findings and/or request additional imaging if the first skeletal survey was not satisfactory, or no findings could be interpreted despite the high index of suspicion. Furthermore, it has been demonstrated that some jurisdictions use the experience of two radiologists for better interpretation of the findings, which can help formulate evidence $(23,24)$. In this context, a previous investigation reported that repeated radiological skeletal surveys are recommended in cases where the first one did not show adequate findings, or when the interpreted findings were abnormal or inconsistent with those suspected based on the clinical information and history of the abused child. Such follow-up procedures were reported to increase the probability of the detection of occult fractures that were not detectable during the initial survey, can effectively estimate the age of the detected fracture, and can significantly quantify the indeterminate findings $(8,25)$. This was further demonstrated in 2013 when a large prospective investigation indicated that $21.5 \%$ of their included cases benefited from performing a follow-up or repeat radiological skeletal surveys. Subsequently, they were able to detect fractures that were not detectable in the initial presentation, and they were also able to facilitate the interpretation of indeterminate findings (26). Omitting images of the pelvis, skull, and spine may also be a sound approach during followup assessment as a result of the demand to reduce the frequency of radiation exposure, in addition to the low probability of getting additional information from these approaches in such situations $(27,28)$.

For adequate complementation of the radiological skeletal surveys, radionuclide bone scans can be utilized. The modality has been reported to have a higher sensitivity than X-rays in detecting subtle shaft fractures of the long bones and rib fractures (Figure 1).

However, evidence shows that X-ray has a higher sensitivity when examining and evaluating a classical metaphyseal lesion. Furthermore, it is well known that skull fractures are not always obtained by bone scan, and therefore, more than one X-ray image should be taken of the skull to adequately view the underlying fracture. Evaluation of the abnormal findings on a bone scan should be additionally undertaken using X-ray for potential detection of an underlying fracture that bone scan cannot reliably detect. In a previous review, the authors concluded that using a single modality of either $\mathrm{X}$-ray or bone scan did not obtain better outcomes when compared to the combined use of both modalities (30). However, evidence also indicates that for the detection of occult trauma, the gold standard in these situations is to perform oblique radiological skeletal surveys on the affected ribs. 


\section{Journal of Healthcare Sciences}
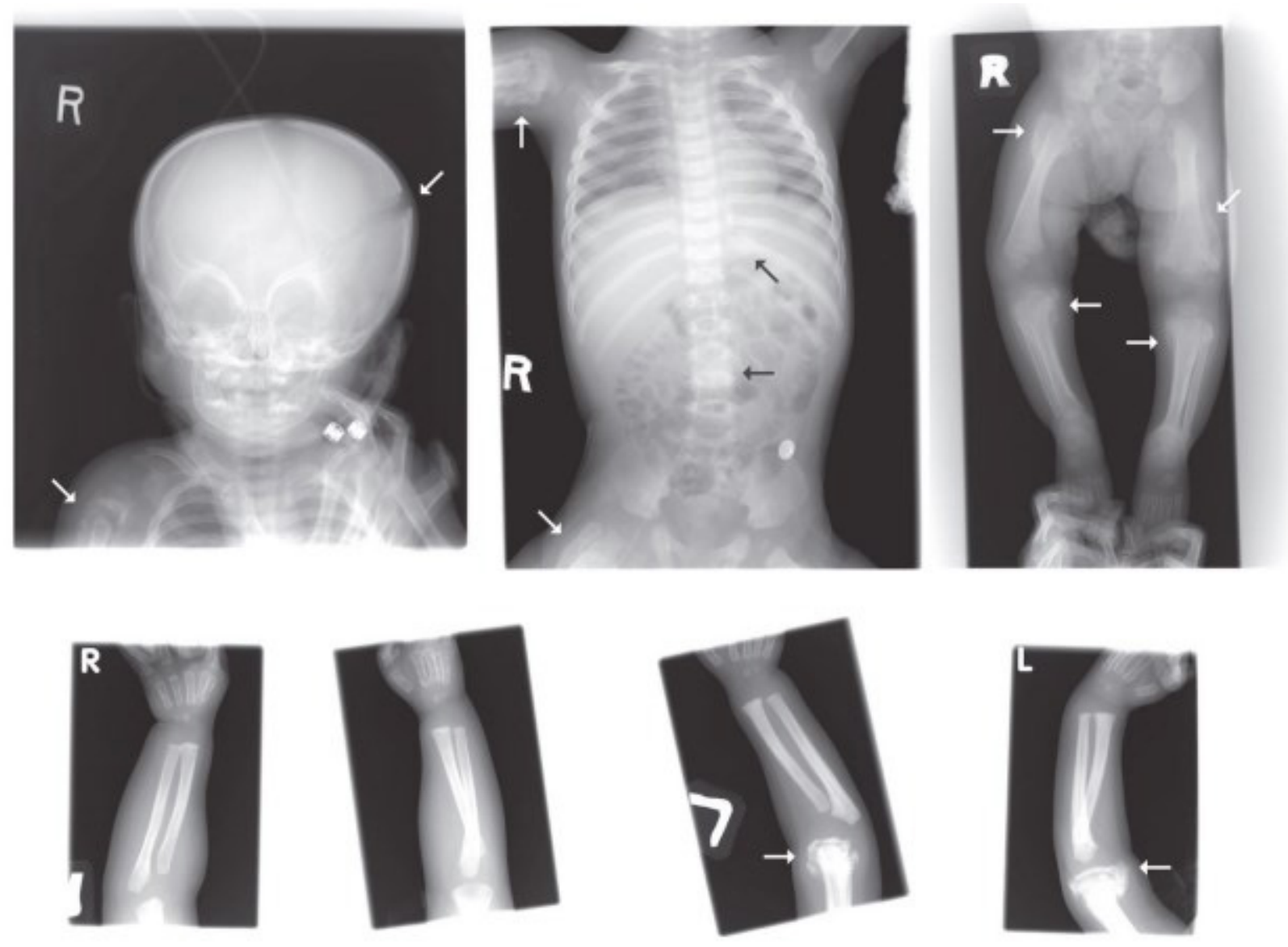

Figure 1. A case of child abuse is indicated by the findings of X-ray (29).

In a separate context, recent investigations show that bedside ultrasound can be used within the emergency department to evaluate and detect long bone and skull fractures $(31,32)$. However, it should be noted that the application of ultrasound in these situations is usually done as an adjacent approach to the routine imaging technique, in addition to being performed secondary to the initial physical assessment of the affected regions. Ultrasound waves are completely reflected by intact cortical bones, and therefore, breaking this pattern is a significant indicator of fractures in the affected area. In cases of children suffering from indeterminate physical abuse, ultrasound imaging can help with the identification of an unsuspected or occult periosteal hematoma, or a bone injury (31). However, it should be noted that this approach should only be conducted under the supervision of an experienced physician to enhance the outcomes and better interpret the findings. Moreover, physicians should be aware that fracture injuries can sometimes be missed by ultrasound imaging techniques. For example, a previous investigation indicated that ultrasound imaging of an area of hematoma of the skull may not obtain the suspected findings in cases when the skull fracture is not located under the skull hematoma (32). Although evidence indicates that bedside ultrasound imaging may offer some validity in detecting skeletal traumas to a potentially similar value to that of bone scans and X-rays, it should not be relied on solely for the evaluation and diagnosis of physical abuse in children. It should also be noted that the interpreted findings should be saved in a medical record in the healthcare settings for additional quality review and legal purposes.

\section{Conclusion}

Conducting radiological skeletal surveys can play a significant role in detecting findings that can indicate the presence of physical abuse. X-ray, bone scan, and bedtime ultrasonography can be used to achieve this with variable sensitivities. However, X-ray remains the most sensitive, and the other two modalities should be used when performing X-ray is not appropriate. Two modalities can also be used at a time for better performance and 


\section{Journal of Healthcare Sciences}

enhanced outcomes. Imaging should be undertaken by at least one experienced radiologist for better interpretation of the findings. Ultimately, radiological skeletal surveys may be optimal for children of approximately 2 years of age, while in children that are $\geq 5$ years old, a case-bycase determination of assessment should be carried out.

\section{Disclosure}

\section{Statement:}

The authors declare no conflict of interest.

\section{Funding:}

None

\section{Ethical Consideration:}

Non applicable.

\section{Data Availability:}

All data are presented within the paper.

\section{Author Contribution:}

All authors equally contributed in conceptualizing, drafting, searching the literature, writing, language editing and proofreading the manuscript.

\section{References:}

1. Kraft JK. (iv) Imaging of non-accidental injury. Orthopaedics and Trauma. 2011;25(2):109-18.

2. Berger RP, Panigrahy A, Gottschalk S, Sheetz M. Effective Radiation Dose in a Skeletal Survey Performed for Suspected Child Abuse. The Journal of pediatrics. 2016;171:310-2.

3. Powell-Doherty RD, Raynor NE, Goodenow DA, Jacobs DG, Stallion A. Examining the role of followup skeletal surveys in non-accidental trauma. American journal of surgery. 2017;213(4):606-10.

4. Hobbs CJ, Bilo RA. Nonaccidental trauma: clinical aspects and epidemiology of child abuse. Pediatric radiology. 2009;39(5):457-60.

5. Patel H, Swinson S, Johnson K. Improving national standards of child protection skeletal surveys: the value of College guidance. Clinical radiology. 2017;72(3):202-6.

6. Nguyen A, Hart R. Imaging of non-accidental injury; what is clinical best practice? Journal of Medical Radiation Sciences. 2018;65(2):123-30.
7. Lindberg DM, Berger RP, Reynolds MS, Alwan RM, Harper NS. Yield of skeletal survey by age in children referred to abuse specialists. The Journal of pediatrics. 2014;164(6):1268-73.e1.

8. Diagnostic imaging of child abuse. Pediatrics. 2009;123(5):1430-5.

9. Jain N. The role of diagnostic imaging in the evaluation of child abuse. British Columbia Medical Journal. $2015 ; 57(8)$

10. Sty JR, Starshak RJ. The role of bone scintigraphy in the evaluation of the suspected abused child. Radiology. 1983;146(2):369-75.

11. Pickett WJ, Faleski EJ, Chacko A, Jarrett RV. Comparison of radiographic and radionuclide skeletal surveys in battered children. Southern medical journal. 1983;76(2):207-12.

12. Mandelstam SA, Cook D, Fitzgerald M, Ditchfield MR. Complementary use of radiological skeletal survey and bone scintigraphy in detection of bony injuries in suspected child abuse. Arch Dis Child. 2003;88(5):38790; discussion -90 .

13. Offiah A, van Rijn RR, Perez-Rossello JM, Kleinman PK. Skeletal imaging of child abuse (non-accidental injury). Pediatric radiology. 2009;39(5):461-70.

14. Conway JJ, Collins M, Tanz RR, Radkowski MA, Anandappa E, Hernandez R, et al. The role of bone scintigraphy in detecting child abuse. Seminars in nuclear medicine. 1993;23(4):321-33.

15. Haase GM, Ortiz VN, Sfakianakis GN, Morse TS. The value of radionuclide bone scanning in the early recognition of deliberate child abuse. J Trauma. 1980;20(10):873-5.

16. Merten DF, Radkowski MA, Leonidas JC. The abused child: a radiological reappraisal. Radiology. $1983 ; 146(2): 377-81$

17. Stöver B. [Diagnostic imaging in child abuse]. Der Radiologe. 2007;47(11):1037-48; quiz 49.

18. Kleinman PK. Diagnostic imaging of child abuse: Cambridge University Press; 2015.

19. Kleinman PK, Marks SC, Jr., Nimkin K, Rayder SM, Kessler SC. Rib fractures in 31 abused infants: postmortem radiologic-histopathologic study. Radiology. 1996;200(3):807-10.

20. Worn MJ, Jones MD. Rib fractures in infancy: establishing the mechanisms of cause from the injuries-a literature review. Medicine, science, and the law. 2007;47(3):200-12. 


\section{Journal of Healthcare Sciences}

21. Barsness KA, Cha ES, Bensard DD, Calkins CM, Partrick DA, Karrer FM, et al. The positive predictive value of rib fractures as an indicator of nonaccidental trauma in children. J Trauma. 2003;54(6):1107-10.

22. Strouse PJ, Owings CL. Fractures of the first rib in child abuse. Radiology. 1995;197(3):763-5.

23. Hulson OS, van Rijn RR, Offiah AC. European survey of imaging in non-accidental injury demonstrates a need for a consensus protocol. Pediatric radiology. 2014;44(12):1557-63.

24. Offiah AC, Adamsbaum C, van Rijn RR. ESPR adopts British guidelines for imaging in suspected non-accidental injury as the European standard. Pediatric radiology. 2014;44(11):1338.

25. Kleinman PK, Nimkin K, Spevak MR, Rayder SM, Madansky DL, Shelton YA, et al. Follow-up skeletal surveys in suspected child abuse. AJR American journal of roentgenology. 1996;167(4):893-6.

26. Harper NS, Eddleman S, Lindberg DM. The utility of follow-up skeletal surveys in child abuse. Pediatrics. 2013;131(3):e672-8.

27. Harlan SR, Nixon GW, Campbell KA, Hansen K, Prince JS. Follow-up skeletal surveys for nonaccidental trauma: can a more limited survey be performed? Pediatric radiology. 2009;39(9):962-8.
28. Sonik A, Stein-Wexler R, Rogers KK, Coulter KP, Wootton-Gorges SL. Follow-up skeletal surveys for suspected non-accidental trauma: can a more limited survey be performed without compromising diagnostic information? Child abuse \& neglect. 2010;34(10):804-6.

29. Chuang Y-W, Hsu C-C, Chang C-C, Lin C-Y, Chu $\mathrm{H}-\mathrm{L}$, Huang Y-F, et al. Multiple Bony Injuries on Bone Scan in a Case of Unsuspected Child Abuse. Case Reports in Medicine. 2017;2017:3015941.

30. Kemp AM, Butler A, Morris S, Mann M, Kemp KW, Rolfe K, et al. Which radiological investigations should be performed to identify fractures in suspected child abuse? Clinical radiology. 2006;61(9):723-36.

31. Warkentine FH, Horowitz R, Pierce MC. The use of ultrasound to detect occult or unsuspected fractures in child abuse. Pediatric emergency care. 2014;30(1):43-6.

32. Rabiner JE, Friedman LM, Khine H, Avner JR, Tsung JW. Accuracy of point-of-care ultrasound for diagnosis of skull fractures in children. Pediatrics. 2013;131(6):e1757-64. 